\title{
Ideal Magnetofluid Turbulence in Two Dimensions
}

\author{
Richard Jordan ${ }^{1.3}$ and Bruce Turkington ${ }^{2}$
}

\author{
Receiced December 18, 1995; find October 24. 1996
}

\begin{abstract}
A continuum model of coherent structures in two-dimensional magnetohydrodynamic turbulence is developed. These structures are macroscopic states which persist among the turbulent microscopic fluctuations. typically as magnetic islands with llow. They are modeled as statistical equilibrium states for the nondissipative dynamics, which conserves energy and families of cross-helicity and tlux integrals. The model predicts that from a given initial state an ideal magnetofluid will evolve into a linal state having steady mean magnetic and velocity fields, and Gaussian local fluctuations in these fields. Excellent qualitative and quantitative agreement is found with the known results of direct numerical simulations. A rigorous justification of the theory is also provided, in the sense that the continuum model is derived from a lattice model in a fixedvolume, small-spacing limit. This construction uses the discrete Fourier transform to link the discretization of $x$-space with the truncation of $k$-space. Under the ergodic hypothesis and a separation-ol-scales hypothesis. the lattice model is delined by a mean-field approximation to the Gibbs measure on the discretized phase space. A concentration property shows that this measure is equivalent to the microcanonical measure in the continuum limit.
\end{abstract}

KEY WORDS: Magnetohydrodynamics; turbulence; coherent structure; statistical equilibrium; maximum entropy; discrete Fourier translorm.

\section{INTRODUCTION}

A conspicuous feature of high-Reynolds-number magnetohydrodynamics (MHD) is the formation of long-lived, large-scale organized states, or coherent structures, amid small-scale turbulent fluctuations. This organization

\footnotetext{
'Center for Nonlinear Analysis, Department of Mathematics, Carnegie Mellon University, Pittsburgh. Pennsylvania 15213.

${ }^{2}$ Department of Mathematics and Statistics, University of Massachusetts. Amherst, Massachusetts 01003 .

${ }^{3}$ Current address: Department of Mathematics. University of Michigan, Ann Arbor, Michigan 48109.
} 
phenomenon is clearly exhibited by two-dimensional systems such as occur in the plane perpendicular to a strong magnetic field. ${ }^{(4.24)}$ Indeed, direct numerical simulations ${ }^{15} 7,31.211$ show that a freely evolving, slightly dissipative 2D magnetofluid relaxes toward a state in which the magnetic and velocity fields exhibit small-scale local fluctuations around a steady mean state that varies on the large spatial scale. The coherent structure defined by these mean fields typically assumes the form of one or more magnetic islands, generally with flow. A similar phenomenon occurs in 2D hydrodynamics at high Reynolds number. In that setting, the coherent structure usually consists of a steady vortex flow that persists in the midst of smallscale vorticity fluctuations. ${ }^{126.81}$ Unlike nonmagnetic hydrodynamic turbulence, however, MHD turbulence is expected to be qualitatively similar in two and three dimensions.

These coherent structures in fluid or plasma turbulence can be modeled as statistical equilibrium states associated with the conservative (nondissipative) dynamics governing the system. Such an ideal model, in which the effects of fluid viscosity and electrical resistivity are ignored, is justified whenever the magnetic and kinetic Reynolds numbers are sufficiently large. Indeed, the organized states in question vary on a spatial scale comparable to the domain size, and form on a temporal scale that is short compared to the corresponding dissipation time. Moreover, the smallscale local fluctuations attached to them are also controlled by the ideal dynamics, since they occur in some inertial range of scales. While the behavior of cascades and other transfer processes cannot be captured in such a statistical equilibrium model, the organized state itself can be characterized by the general principle that entropy be maximized subject to the constraints dictated by the ideal dynamics. ${ }^{11.21}$

Two fundamental difficulties are confronted, however, when the 2D MHD system is placed in this theoretical framework. First, the continuum system is infinite-dimensional, and hence some kind of discretization is required to make it amenable to a probabilistic treatment. Second, the ideal dynamics conserves not only energy, but also two infinite families of cross-helicity and flux integrals, which give the system its distinctive character. The compatible resolution of these difficulties accordingly becomes a key point in the construction of a statistical equilibrium model. As is explained in the review by Kraichnan and Montgomery, ${ }^{(22)}$ models of this kind therefore tend to fall into two categories: " $k$-space models" and " $x$-space models." While these categories principally refer to whether the system is truncated in wavenumber space or discretized in physical space, they also relate to how the conserved quantities are included in the model.

The $k$-space model for 2D MHD turbulence, indicated by Lee, ${ }^{\{23\}}$ was developed by Fyfe and Montgomery. ${ }^{(13)}$ This statistical equilibrium theory 
is based upon a canonical measure for a truncated spectral representation of the governing equations. While this model captures some of the essential features of the turbulent state, it accounts only for the purely quadratic invariants of the ideal dynamics, ignoring the invariants that are not easily expressible in a spectral form. As a result of this simplification, it yields equilibrium distributions whose mean magnetic and velocity fields vanish identically, and hence it fails to predict a (nontrivial) coherent structure. ${ }^{(34)}$ Instead, it contains finite energy fluctuations in the lowest mode, which in the continuum limit contradicts the equivalence-of-ensembles postulate on which the theory rests. These defects of the $k$-space approach are removed in recent work by Gruzinov and Isichenko, ${ }^{(14.17)}$ who use a formal asymptotic analysis to build a steady mean state into the model. They obtain a meaningful continuum limit by appropriately rescaling the inverse temperature-like parameters in the canonical ensemble with the number of spectral modes. Their analysis, which ultimately relies on a separationof-scales assumption, shows how the conserved integrals (energy, crosshelicity, and flux) partition between the large-scale mean state and the small-scale fluctuations.

The $x$-space approach is taken by Montgomery et $a l^{(29)}$ Their model uses a field-line discretization of the current density and vorticity, combined with an information-theoretic entropy. ${ }^{(18)}$ It yields equilibrium equations for the most probable state by maximizing this entropy subject to constraints on the classical conserved quantities. The predicted macroscopic state is, however, not necessarily a steady solution of the MHD equations. This property, along with some arbitrariness in the construction of the maximum entropy principle itself, suggests that the underlying $x$-space discretization is too crude. A different $x$-space approach is proposed by the present authors. ${ }^{(37)}$ Our model draws upon the ideas introduced by Robert $^{(32)}$ in the context of $2 \mathrm{D}$ hydrodynamic turbulence. The major innovation of this approach is the use of an $x$-parametrized probability measure (or Young measure) to provide the macroscopic description of the organized state. In the theory of $2 \mathrm{D}$ vortex structures, due independently to Robert et al. ${ }^{(32.33)}$ and Miller et al., ${ }^{(27.28)}$ the macrostate represents a probability distribution on the values of the fluctuating vorticity field near each spatial point. (Methods of a similar nature were used earlier by Lynden-Bell in a heuristic statistical model of violent relaxation in stellar systems, ${ }^{(25)}$ and essentially all such statistical theories of fluid turbulence have their roots in the pioneering work of Onsager ${ }^{(30)}$ on the statistical mechanics of fluid vortices.) In an analogous manner, our theory of $2 \mathrm{D}$ magnetofluid turbulence makes use of an $x$-parametrized joint probability distribution on the values of the fluctuating magnetic and velocity fields. Such a description is valid under the hypothesis that the local fluctuations 
occur on an infinitesimal scale at each point, and that they are uncorrelated between distinct points. This separation-of-scales hypothesis leads directly to a continuum $x$-space model governed by a natural constrained maximum entropy principle. As is demonstrated in Ref. 37 the most probable state is a steady solution of the 2D MHD equations, and the local fluctuations are Gaussian. These basic results of our $x$-space model agree with those obtained by Gruzinov and Isichenko ${ }^{(17)}$ from their $k$-space model.

In the present paper we give a complete theory of coherent structures in ideal 2D MHD turbulence, using a synthesis of the $x$-space and $k$-space methods. We examine the continuum model enunciated in our earlier paper $^{(37)}$ from two points of view. First, we analyze its predictions and compare them with some known results of direct numerical simulations. Second, we justify its formulation by deriving it as the convergent limit of a lattice model. This derivation, along with the construction of the lattice model, is perhaps the main contribution of the paper. We construct the appropriate lattice model (for doubly periodic boundary conditions) using the discrete Fourier transform, which allows us to exploit the relationship between truncation in $k$-space and discretization in $x$-space, and thereby to clarify the separation-of-scales hypothesis. Our lattice model is defined by a mean-field approximation to the classical Gibbs measure for the discrete system. In essence, our mean-field theory defines the simplest invariant measure on the discrete phase space that respects both the separation-ofscales condition and the complete family of dynamical invariants.

The paper is organized as follows. In Section 2, we formulate the $x$-space continuum model in a general domain. The constrained maximum entropy principle that defines the model is developed in this section from intuitive, physical considerations. In Section 3, we analyze the statistical equilibrium states governed by the continuum model as thoroughly as possible, without resorting to numerical methods. This section extends the analysis published by Jordan ${ }^{(19)}$ for the special case in which the family of cross-helicity integrals is reduced to the classical quadratic cross-helicity. In Section 4, we construct the lattice model and use it to give a rigorous derivation of the continuum model formulated heuristically in Section 2.

\section{IDEAL MAGNETOFLUID TURBULENCE}

\subsection{Microscopic Dynamics.}

The equations governing ideal magnetohydrodynamics (MHD) are expressible in a normalized, dimensionless form as ${ }^{(4.24)}$ 


$$
\begin{aligned}
& \frac{\partial B}{\partial t}=\nabla \times(V \times B), \quad \nabla \cdot B=0 \\
& \frac{\partial V}{\partial t}=V \times(\nabla \times V)+(\nabla \times B) \times B-\nabla P, \quad \nabla \cdot V=0
\end{aligned}
$$

where $B$ is the magnetic induction field, $V$ is the fluid velocity field, and $P=p+\frac{1}{2} V^{2}$ is the total pressure head. The fluid is incompressible with mass density normalized to unity. The conducting and flowing medium is ideal in the sense that its resistivity and viscosity are both ignored. Under these conditions the induced electric field is given by $E=-V \times B$, and therefore it does not enter into the governing equations. Similarly, the pressure head $P$ is determined instantaneously by $B$ and $V$ in response to the incompressibility constraint. For these reasons, the state of the magnetofluid is completely described by the vector field

$$
Y(x, t):=(B, V)
$$

which we shall call the field-flow state.

We are concerned exclusively with purely two-dimensional systems. We let the domain $D \subset R^{2}$ be the crosssection of the spatial region $D \times R$ occupied by the magnetofluid, and we assume that the field-flow state has the form $Y=\left(B_{1} e_{1}+B_{2} e_{2}, V_{1} e_{1}+V_{2} e_{2}\right)$ in which the components are functions of $x=\left(x_{1}, x_{2}\right) \in D$. For the sake of simplicity, we take the domain boundary $\partial D$ to be finite and regular. On $\partial D$ we impose the ideal boundary conditions $n \cdot B=0, n \cdot V=0$, with $n$ normal to the boundary. Alternatively, in Section 4 we consider periodic boundary conditions on a rectangle $D$ with fundamental periods $L_{1}$ and $L_{2}$ in $x_{1}$ and $x_{2}$, respectively. In the periodic case, we also require that $Y$ averages to zero over $D$.

The induction Eq. (2.1) implies that the magnetic lines of force are frozen into the mass flow (the $B$-lines are advected by $V$ ) and that the magnetic flux of any tube composed of these lines is conserved by the flow (the flux of each $B$-tube is constant in time). In two dimensions these central properties of ideal MHD are expressed succinctly by the equation

$$
\frac{\partial \psi}{\partial t}+V \cdot \nabla \psi=0
$$

for the flux function $\psi$, which is defined by $B=\nabla \times \psi e_{3}$. The scalar advection Eq. (2.4) is equivalent to the primitive Eqs. (2.1). In an analogous 
fashion, the primitive momentum Eqs. (2.2) are reducible to the scalar equation

$$
\frac{\partial \omega}{\partial t}+V \cdot \nabla \omega=B \cdot \nabla j
$$

in which $\omega=e_{3} \cdot \nabla \times V$ is the vorticity and $j=e_{3} \cdot \nabla \times B$ is the current density. In contrast to the nonmagnetic situation, where the vorticity is rearranged by the flow it induces, the evolution Eq. (2.5) contains both advection and intensification, which arises from the $J \times B$ body force term in (2.2). These flux-vorticity equations for ideal MHD in two dimensions are often useful in deriving properties of solutions. Moreover, they are intimately connected to the (noncanonical) Hamiltonian structure of the conservative dynamical system $(2.1)-(2.2) .^{(15)}$ This structure is manifest once the stream function $\phi$ is introduced by $V=\nabla \times \phi e_{3}$. The flux-vorticity equations can then be written in the form

$$
\frac{\partial \psi}{\partial t}+\partial(\psi, \phi)=0, \quad \frac{\partial \omega}{\partial t}+\partial(\omega, \phi)=\partial(j, \psi)
$$

using the canonical bracket on $R^{2}$ defined by $\partial(\psi, \phi)=e_{3} \cdot \nabla \psi \times \nabla \phi$.

For use throughout the paper, we introduce a notation for the linear operators that mediate between the primitive vector fields $B$ and $V$ and the derived scalar fields $\psi, j$ and $\phi, \omega$. This is necessary because the statistical description of turbulent field-flow utilizes the primitive fields, while the conserved quantities and equilibrium equations involve the derived scalar fields. We let "curl" and "Curl" be defined by

$$
\operatorname{curl} B:=e_{3} \cdot \nabla \times B, \quad \text { Curl } \psi:=\nabla \times \psi e_{3}
$$

for any vector field $B$ and any scalar field $\psi$. We note that curl acts on a vector and produces a scalar, while Curl acts on a scalar and produces a vector. These operators are adjoints in the sense that

$$
\int_{D} \psi \operatorname{curl} \widetilde{B} d x=\int_{D} \operatorname{Curl} \psi \cdot \widetilde{B} d x
$$

for any $\psi$ and $\widetilde{B}$ satisfying the ideal boundary conditions. We especially need the inverses of curl and Curl. Let $G$ be the Green operator for the boundary-value problem

$$
-\Delta \psi=j \text { in } D, \quad \psi=0 \text { on } \partial D
$$


so that the solution is expressed $\psi=G j$. Using the identity $-\Delta=$ curl Curl, we obtain the inverse operators

$$
\operatorname{curl}^{-1} j:=\operatorname{Curl}(G j), \quad \operatorname{Curl}^{-1} B:=G(\operatorname{curl} B)
$$

for any scalar field $j$ and any vector field $B$. We note that these formulas determine the inverse operators completely, without further integrability or gauge conditions. Indeed, $B=\operatorname{curl}^{-1} j$ is solenoidal $(\nabla \cdot B=0$ in $D$ and $n \cdot B=0$ on $\partial D$ ) for an arbitrary density $j$, and $\psi=\mathrm{Curl}^{-1} B$ defines the flux function for the solenoidal part of an arbitrary field $B$. The identity (2.7) translates into the statement that $\mathrm{curl}^{-1}$ and $\mathrm{Curl}^{-1}$ are adjoints:

$$
\int_{D}\left(\operatorname{curl}^{-1} B\right) \tilde{J} d x=\int_{D} B \cdot \operatorname{curl}^{-1} \tilde{J} d x
$$

We use this reciprocal identity for several calculations in the sequel.

The complete family of conserved quantities for two-dimensional MHD is ${ }^{(39)}$

$$
\begin{aligned}
& E=\int_{D} \frac{1}{2}\left(|B|^{2}+|V|^{2}\right) d x \\
& F_{i}=\int_{D} f_{i}(\psi) d x \quad\left(\psi=\operatorname{Curl}^{-1} B\right) \\
& K_{i}=\int_{D} \omega f_{i}(\psi) d x=\int_{D} B \cdot V f_{i}^{\prime}(\psi) d x \quad(\omega=\operatorname{curl} V)
\end{aligned}
$$

The total energy integral $E=E_{\text {mig }}+E_{\text {kin }}$ coincides with the Hamiltonian functional for the system. The families of conserved quantities $F_{i}$ and $K_{i}$, indexed by $i$, are Casimir functionals that arise from the degeneracy of the (noncanonical) Hamiltonian structure. ${ }^{(15)}$ In both of these families, $f_{i}(\psi)$ denotes an arbitrary (regular) real function defined on the range of the flux function $\psi(x, t)$. This range is invariant under evolution by (2.4). It suffices to let $i$ be a continuous index running over some interval $I$, that parametrizes the flux levels $\psi=\sigma_{i}$ realized by all the magnetic surfaces in $D$. The physical meaning of the Casimir functionals is revealed by choosing $f_{i}(\psi)=1_{\left\{\psi>\sigma_{i}\right\}}$, the unit step function on the interior of the magnetic surface $\psi=\sigma_{i}$. Then $F_{i}$ equals the mass and $K_{i}$ equals the circulation inside the flux tube with index $i \in I$. That these quantities are constants of the motion can be verified directly from the flux and vorticity Eqs. (2.4) and (2.5). We refer to the integrals $F_{i}$ and $K_{i}$, in which $f_{i}(\psi)$ is a general (regular) real function, as the generalized flux and generalized cross-helicity, 
respectively. The second form of $K_{i}$ noted in (2.13) follows upon an integration by parts. The classical cross-helicity integral results from the particular choice $f_{i}^{\prime}(\psi)=1$. (Here and throughout the sequel, prime denotes $d / d \psi$.) We expect that $E, F_{i}, K_{i}$ exhaust the set of global conserved integrals for the ideal dynamics, apart from those that arise from special spatial symmetries depending on the domain geometry and boundary conditions.

Both for analytical and numerical reasons, it is convenient to approximate the continuously infinite families of generalized flux and crosshelicity integrals by the linear combination of a finite number of such integrals. Accordingly, we choose some finite basis of functions $f_{i}(\sigma)$, $i=1, \ldots, h$, with argument $\sigma \in R$, having suitable regularity and growth properties, and we retain only the corresponding integrals $F_{i}$ and $K_{i}$ for $i=1, \ldots, h$. We then retrieve an arbitrary $f(\psi)$ in the approximate sense that $f(\psi) \approx \sum_{i=1}^{h} c_{i} f_{i}(\psi)$ for an appropriate set of constants $c_{i}$. Even for a fairly small $h$, we expect this approximation to be quite accurate, owing to the natural regularity of $\psi .^{(12,38)}$ We therefore adopt it throughout the sequel to ease the technical complications associated with continuously infinite families of constraints.

\subsection{Macroscopic Description}

The evolution of an ideal magnetofluid is turbulent in the sense that the field-flow state $Y=Y(x, t)$ spontaneously develops finite-amplitude fluctuations on small scales, even when its initialization $Y^{0}=Y(x, 0)$ varies smoothly over the domain. This behavior of the deterministic governing Eqs. (2.1) $-(2.2)$ is strongly supported by direct numerical simulations, which necessarily pertain to a slightly dissipative perturbation of the ideal equations. ${ }^{(4-7.31 .21)}$ An extrapolation of these simulations to the ideal limit suggests that as time proceeds the fluctuations of $Y$ reside on smaller and smaller scales around each point $x$, and that the correlations between these fluctuations at two separated points $x$ and $x^{\prime}$ become weaker and weaker. In constructing a model of the final state of ideal turbulence, therefore, we are led to a description in which the fluctuations of $Y=(B, V)$ have finite variance, occur on an infinitesimal scale around each point $x$, and are uncorrelated between distinct points $x$ and $x^{\prime}$. This turbulent state is not homogeneous, however, as it exhibits a nonzero mean field-flow $\bar{Y}=(\bar{B}, \bar{V})$ that emerges on the macroscopic scale among the microscopic fluctuations. Indeed, high-resolution computational studies clearly demonstrate that a long evolution produces a coherent structure that varies on the scale of the domain size. ${ }^{(5,34,21)}$

On the basis of these qualitative observations, we are now able to formulate our continuum model of ideal turbulence. We defer a rigorous 
derivation of the model from the principles of equilibrium statistical mechanics until Section 4. Below we are content to derive a statement of the maximum entropy principle governing the model from intuitive, physical considerations.

We base our continuum model on two postulates: an ergodic hypothesis and a separation-of-scales hypothesis. The ergodic hypothesis recognizes that the long-time average of the microscopic dynamics can be represented macroscopically by an ensemble average, which is defined by a statistical equilibrium measure. The separation-of-scales hypothesis implies that this measure can be factored into $x$-local probability measures on the fluctuating microstate $Y(x)$. In other words, we express the microstate $Y=\bar{Y}+Y^{\prime}$ in terms of the mean $\bar{Y}$ and the fluctuations $Y^{\prime}$, and we assume that $\bar{Y}$ varies on the scale of the domain, while $Y^{\prime}$ consists of finite variance, uncorrelated fluctuations localized at each point of the domain. Together these two hypotheses allow us to introduce a simple macroscopic description of the ideal MHD system. Namely, we consider the probability distribution $p_{. x}(d y)$ on the values $y \in R^{4}$ of the microstate $Y(x, t)$ at each point $x \in D$. This $x$-parametrized probability measure is defined by the property that

$$
\int_{, I} d x \int_{: ! \prime} p_{x}(d y)=\lim _{T \rightarrow \infty} \frac{1}{T} \int_{0}^{T}|\{x \in \mathscr{X}: Y(x, t) \in \mathscr{Y}\}| d t
$$

for all measurable sets $\mathscr{X} \subset D$ and $\mathscr{Y} \subset R^{4}$; here, $|\mathscr{S}|$ denotes the 2-volume of a subset $\mathscr{S}$ of $D$. This definition means that for any spatial cell $d x$ around $x$ and any cell $d y$ in the range of $Y$, the probability $p_{x}(d y)$ equals the average over $d x$ of the statistical frequency with which $Y$ takes values in $d y$; in symbols,

$$
p_{. x}(d y)=\lim _{|d x| \rightarrow 0} \lim _{T \rightarrow \infty} \frac{1}{T} \int_{0}^{T} \frac{\left|x^{\prime} \in d x: Y\left(x^{\prime}, t\right) \in d y\right|}{|d x|} d t
$$

Alternatively, $p_{x}(d y)$ can be interpreted as the time (or ensemble) average of the local volume fraction around $x$ over which $Y$ lies in $d y$. The macrostate $p_{x}(d y)^{\circ}$ furnishes a macroscopic description of the field-flow state, which complements the microscopic description inherent in $Y(x, t)$; it constitutes a "coarse-grained" version of the "fine-grained" field-flow state $Y=(B, V)$. Our separation-of-scales hypothesis permits us to pose the continuum model in terms of this single-point, local probability distribution $p_{x}(d y)$ rather than a joint probability distribution over many points $x^{(1)}, \ldots, x^{(N)}$, with $N \rightarrow \infty$ in the continuum limit. 
Henceforth we shall use the probability density $\rho_{. x}(y)$ of $p_{x}(d y)$ with respect to the 4-volume element $d y$ as the macrostate for the system. In terms of $\rho_{x}(y)$ the defining identity (2.14) can be put in the simple form

$$
\rho_{x}(y)=\lim _{T \rightarrow-\infty} \frac{1}{T} \int_{0}^{T} \delta(y-Y(x, t)) d t
$$

where $\delta$ is the unit delta function on $R^{4}$, and the convergence is in the weak sense with respect to both $x$ and $y$. This formal statement means that

$$
\int_{D} \int_{R^{+}} a(x, y) \rho_{x}(y) d x d y=\lim _{T \rightarrow \infty} \frac{1}{T} \int_{0}^{T} d t \int_{D} a(x, Y(x, t)) d x
$$

holds for all bounded, continuous test functions $a(x, y)$ on $D \times R^{4}$. This identity is equivalent to the ergodic hypothesis, which equates the time averages with the ensemble averages of any functional having the form $A(Y)=\int_{1} a(x, Y(x)) d x$. This special form of the test functionals $A$ is dictated by the separation-of-scales hypothesis. In postulating the weak convergence of the empirical measures in (2.16), we also require that they satisfy a uniform integrability condition ${ }^{(3)}$ which ensures the convergence of their moments. In particular, this mild condition implies that the mean and variance of $Y$ at $x \in D$, namely,

$$
\bar{Y}(x):=\int_{R^{4}} y \rho_{. x}(y) d y, \quad \operatorname{var} Y(x):=\int_{R^{4}}|y-\bar{Y}(x)|^{2} \rho_{x}(y) d y
$$

are achieved as limits in (2.17) by taking $a=\xi(x) y$ and $a=\xi(x)|y-\bar{Y}(x)|^{2}$, respectively, for all test functions $\zeta(x)$ on $D$.

The various scalar and vector fields participating in the microscopic description of the ideal MHD system have distinctly different local statistical properties. On the one hand, the variance of $Y=(B, V)$ is expected to be finite in the continuum model. This fact is fundamental to our construction of the macrostate $\rho$. On the other hand, the potentials $\psi=\mathrm{Curl}^{-1} B$ and $\phi=\mathrm{Curl}^{-1} V$ are expected to have infinitesimally small variance in the continuum limit, by virtue of the separation-of-scales hypothesis. Indeed, the insensitivity of $\psi$ to the local fluctuations in $B$, which are uncorrelated at different points, can be seen heuristically by applying the law of large numbers ${ }^{(3)}$ to the integral representation

$$
\psi(x)=\int_{D} \operatorname{Curl}_{x} g\left(x, x^{\prime}\right) \cdot B\left(x^{\prime}\right) d x^{\prime}
$$


where $g$ is the Green function for $-\Delta$ on $D$. As a means of formulating the continuum model, therefore, we assert that at each $x \in D$

$$
\bar{\psi}(x)=\operatorname{Curl}^{-1} \bar{B}, \quad \operatorname{var} \psi(x)=0
$$

A rigorous estimation of the variance of $\psi$ constitutes a crucial part of the analysis given in Section 4. The analogous expressions also hold for $\phi(x)$. Thus, the flux function and stream function coincide with their means. By a similar reasoning, we see that $j$ and $\omega$ are expected to fluctuate with unbounded variances in the continuum formulation, and accordingly their local statistical properties are not quantified in the continuum model.

The statistical equilibrium macrostate $\rho$ is taken to be the entropymaximizing state subject to the constraints imposed by the conservation of energy, flux, and cross-helicity. In other words, the system relaxes on the macroscopic scale into the most probable state satisfying the given initial values of the conserved quantities. The entropy functional compatible with the separation-of-scales hypothesis is

$$
S(\rho)=-\int_{D} \int_{R^{+}} \rho_{x}(y) \log \rho_{x}(y) d x d y
$$

This classical Boltzmann-Gibbs-Shannon formula(1,2) admits the usual interpretation as the logarithm of the number of microscopic realizations of the macrostate $\rho$. Alternatively, it is identical with the Kullback-Liebler entropy "'l' the measure $p_{x}(d y) d x$ on $D \times R^{4}$ of relative to the spatially homogeneous measure $d x d y$. Its form is dictated by two properties of the ideal turbulence: (1) as an integral in $x$, it imposes independence on the fluctuations at separate points in $D ;(2)$ as an integral in $y$, it puts uniform weight on the entire range $R^{4}$ of the fluctuations. The full justification of these postulated properties rests on the Liouville property of the underlying dynamics, as is shown in Section 4.

The entropy serves as the objective functional in the maximum entropy principle which determines the statistical equilibrium state. The constraints in this principle, which give the system its special character, are imposed on the following functionals of $\rho$ :

$$
\begin{aligned}
& E(\rho)=\int_{D} \int_{R^{4}} \frac{1}{2}\left(|b|^{2}+|v|^{2}\right) \rho_{x}(y) d x d y \\
& F_{i}(\rho)=\int_{D} f_{i}(\bar{\psi}(x)) d x \\
& K_{i}(\rho)=\int_{D} \int_{R^{4}} b \cdot v f_{i}^{\prime}(\bar{\psi}(x)) \rho_{x}(y) d x d y
\end{aligned}
$$


in which $b$ and $v$ each run over $R^{2}$ with $y=(b, v)$, and $\bar{\psi}$ is defined by (2.19). These expressions are derived by applying the time-averaging identity (2.17) along with the property (2.19) to the conserved quantities (2.11)-(2.13) for the microscopic dynamics. Again, the rigorous derivation is delayed until Section 4.

With the objective and constraint functionals in hand, we finally arrive at the maximum entropy principle for the statistical equilibrium state $p$ :

$$
S(\rho) \rightarrow \max \text { over } E(\rho)=E^{0}, \quad F_{i}(\rho)=F_{i}^{0}, \quad K_{i}(\rho)=K_{i}^{0}
$$

where the constraint values $E^{0}, F_{i}^{0}$, and $K_{i}^{0}$ are derived from a given microstate $Y^{0}$. Of course, $\rho$ must also satisfy the positivity condition, $\rho_{x}(y) \geqslant 0$, and the normalization constraint, $\int_{R^{4}} \rho_{x}(y) d y=1$ for all $x \in D$. This constrained optimization problem completely specifies the continuum model. Its solution $\rho$ represents the final state that emerges after a turbulent evolution from a given initial state.

\section{PROPERTIES OF STATISTICAL EQUILIBRIUM STATES}

\subsection{Analysis of Solutions}

The equilibrium equation satisfied by the solution to the maximum entropy problem is obtained from the Lagrange multiplier rule

$$
\delta S=\beta \delta E+\sum_{i} \alpha_{i} \delta F_{i}+\sum_{i} \gamma_{i} \delta K_{i}
$$

which holds on the subspace of variations $\delta \rho$ satisfying the local normalization $\int \delta \rho_{x}(y) d y=0$. Here $\delta \Phi$ denotes the first variation of a functional $\Phi(\rho)$ with respect to $\rho$. The multipliers $\beta, \alpha_{i}, \gamma_{i}$ for the constraints are analogous to the "inverse temperature" in usual statistical mechanics. (1. 2) We calculate the functional derivatives appearing in (3.1) to be

$$
\begin{aligned}
& \delta S=-\iint\left[\log \rho_{x}(y)\right] \delta \rho_{x}(y) d x d y \\
& \delta E=\iint \frac{1}{2}\left(|b|^{2}+|v|^{2}\right) \delta \rho_{x}(y) d x d y \\
& \delta E=\iint b \cdot \operatorname{curl}^{-1} f_{i}^{\prime}(\bar{\psi}) \delta \rho_{x}(y) d x d y \\
& \delta K_{i}=\iint b \cdot\left\{f_{i}^{\prime}(\bar{\psi}) v+\operatorname{curl}^{-1}\left[\overline{B \cdot V} f_{i}^{\prime \prime}(\bar{\psi})\right]\right\} \delta \rho_{x}(y) d x d y
\end{aligned}
$$


where each double integral extends over $x \in D$ and $y=(b, v) \in R^{4}$. The calculations (3.2) and (3.3) are straightforward, while (3.4) and (3.5) deserve some explanation. We obtain (3.4) by the following steps:

$$
\begin{aligned}
\delta F_{i} & =\int f_{i}^{\prime}(\bar{\psi}) \delta \bar{\psi} d x \\
& =\int f_{i}^{\prime}(\bar{\psi}) \mathrm{Curl}^{-1} \delta \bar{B} d x \\
& =\int \operatorname{curl}^{-1} f_{i}^{\prime}(\bar{\psi}) \cdot \delta \bar{B} d x \\
& =\iint \operatorname{curl}^{-1} f_{i}^{\prime}(\bar{\psi}) \cdot b \delta \rho_{x}(y) d x d y
\end{aligned}
$$

in which we appeal to the adjoint formula (2.10). We get (3.5) by a similar sequence of steps, which now involve the mean cross-helicity density

$$
\overline{B \cdot V}(x):=\int_{R^{+}} b \cdot v \rho_{x}(y) d y
$$

Upon substitution of these expressions into (3.1), we deduce that the statistical equilibrium macrostate $\rho$ has the local canonical form

$$
\rho_{x}(y)=Z_{x}^{-1} \exp [-\beta q(y ; \lambda(\bar{\psi}), \mu(\bar{\psi}))]
$$

with

$$
Z_{x}=\int_{R^{\mathrm{u}}} \exp [-\beta q(y ; \lambda(\bar{\psi}), \mu(\bar{\psi}))] d y
$$

Here we introduce the quadratic form

$$
\begin{aligned}
q(y ; \lambda, \mu):= & \frac{1}{2}\left(|b|^{2}+|v|^{2}\right) \\
& -b \cdot \operatorname{curl}^{-1} \lambda(\sigma)-b \cdot\left\{\mu(\sigma) v+\operatorname{curl}^{-1}\left[\overline{B \cdot V} \mu^{\prime}(\sigma)\right]\right\}
\end{aligned}
$$

and the profile functions

$$
\lambda(\sigma):=-\beta^{-1} \sum_{i} \alpha_{i} f_{i}^{\prime}(\sigma), \quad \mu(\sigma)=-\beta^{-1} \sum_{i} \gamma_{i} f_{i}^{\prime}(\sigma)
$$


We see immediately that the solution $\rho_{x}(y)$ is a Gaussian density in $y$ for every $x \in D$. We also note that the covariance matrix for these local distributions depends implicitly on the mean field $\bar{\psi}(x)$. Moreover, this dependence is nonlinear and nonlocal, owing to the presence of the (nonlinear) profile functions $\lambda$ and $\mu$ and the (nonlocal) operator curl ${ }^{-1}$.

We extract the mean field-flow $\bar{Y}=(\bar{B}, \bar{V})$ and the fluctuations $Y^{\prime}=Y-\bar{Y}$ from the basic formula (3.7) by introducing the variables $b^{\prime}=b-\bar{B}(x), v^{\prime}=v-\bar{V}(x)$, in terms of which we get the normal form

$$
q=\frac{1}{2}\left(\left|b^{\prime}\right|^{2}+\left|v^{\prime}\right|^{2}\right\rangle-b^{\prime} \cdot v^{\prime} \mu(\bar{\psi})-\frac{1}{2}\left[1-\mu(\bar{\psi})^{2}\right]|\bar{B}|^{2}
$$

and the mean field-flow equations

$$
\begin{aligned}
& \bar{B}=\operatorname{curl}^{-1}\left[\lambda(\bar{\psi})+\overline{B \cdot V} \mu^{\prime}(\bar{\psi})\right]+\mu(\bar{\psi}) \bar{V} \\
& \bar{V}=\mu(\bar{\psi}) \bar{B}
\end{aligned}
$$

The equilibrium macrostate is then given by

$$
\rho_{x}(y)=\left(\frac{\beta}{2 \pi}\right)^{2}\left[1-\mu(\bar{\psi})^{2}\right] \exp \left\{-\frac{\beta}{2}\left[\left|b^{\prime}\right|^{2}+\left|v^{\prime}\right|^{2}-2 \mu(\bar{\psi}) b^{\prime} \cdot v^{\prime}\right]\right\}
$$

after some straightforward manipulations. We now proceed to analyze these equations for the joint Gaussian distribution on $B$ and $V$.

The $4 \times 4$ covariance matrix for $Y$ at $x \in D$ is readily deduced from (3.14), giving

$$
\begin{gathered}
\operatorname{var} B_{1}=\operatorname{var} B_{2}=\operatorname{var} V_{1}=\operatorname{var} V_{2}=\beta^{-1}\left[1-\mu(\bar{\psi})^{2}\right]^{-1} \\
\operatorname{cov}\left(B_{1}, V_{1}\right)=\operatorname{cov}\left(B_{2}, V_{2}\right)=\beta^{-1} \mu(\bar{\psi})\left[1-\mu(\bar{\psi})^{2}\right]^{-1}
\end{gathered}
$$

along with $\operatorname{cov}\left(B_{p}, B_{q}\right)=\operatorname{cov}\left(V_{p}, V_{q}\right)=\operatorname{cov}\left(B_{p}, V_{q}\right)=0$ for $p \neq q$. From these formulas we see that $\mu(\bar{\psi})$ is the magnetic-velocity correlation,

$$
\mu(\bar{\psi})=\operatorname{corr}\left(B_{p}, V_{p}\right):=\frac{\operatorname{cov}\left(B_{p}, V_{p}\right)}{\sqrt{\operatorname{var} B_{p}} \sqrt{\operatorname{var} V_{p}}} \quad(p=1,2)
$$

We observe that these expressions require that $0<\beta<\infty$ and $-1<$ $\mu(\bar{\psi})<1$, with equality in degenerate cases only.

The energy density and the cross-helicity density are given by

$$
\begin{aligned}
\frac{1}{2}\left(\mid \overline{\left.B\right|^{2}}+\overline{|V|^{2}}\right) & =\frac{1}{2}\left(|\bar{B}|^{2}+|\bar{V}|^{2}\right)+2 \beta^{-1}\left[1-\mu(\bar{\psi})^{2}\right]^{-1} \\
\overline{B \cdot V} & =\bar{B} \cdot \bar{V}+2 \beta^{-1} \mu(\bar{\psi})\left[1-\mu(\bar{\psi})^{2}\right]^{-1}
\end{aligned}
$$


using (3.15) and (3.16), respectively. Consequently, the equilibrium expressions for the energy and generalized cross-helicity are

$$
\begin{aligned}
E & =\int \frac{1}{2}\left(|\bar{B}|^{2}+|\bar{V}|^{2}\right) d x+\frac{2}{\beta} \int \frac{1}{1-\mu(\bar{\psi})^{2}} d x \\
K_{i} & =\int \bar{B} \cdot \bar{V} f_{i}^{\prime}(\bar{\psi}) d x+\frac{2}{\beta} \int \frac{\mu(\bar{\psi})}{1-\mu(\bar{\psi})^{2}} f_{i}^{\prime}(\bar{\psi}) d x
\end{aligned}
$$

These expressions exhibit the partitioning of $E$ and $K_{i}$ into mean and fluctuation parts. By contrast, the generalized flux $F_{i}$ resides entirely in the mean.

The mean field-flow Eqs. (3.12)-(3.13) reduce to a single equation for the mean magnetic field, upon eliminating $\bar{V}$ and substituting (3.19) for $\overline{B \cdot V}$ :

$$
\left[1-\mu(\bar{\psi})^{2}\right] \bar{B}=\operatorname{curl}^{-1}\left[\lambda(\bar{\psi})+\mu(\bar{\psi}) \mu^{\prime}(\bar{\psi})\left\{|\bar{B}|^{2}+2 \beta^{-1}\left[1-\mu(\bar{\psi})^{2}\right]^{-1}\right\}\right]
$$

In turn, this equation simplifies to a scalar equation for $\bar{\psi}$, by taking curl:

$$
\bar{\jmath}-\mu(\bar{\psi}) \bar{\omega}=\Lambda(\bar{\psi}):=\lambda(\bar{\psi})+2 \beta^{-1} \mu(\bar{\psi}) \mu^{\prime}(\bar{\psi})\left[1-\mu(\bar{\psi})^{2}\right]^{-1}
$$

We draw the important conclusion that the mean field-flow $(\bar{B}, \bar{V})$ is a steady solution of the ideal MHD Eqs. (2.4)-(2.5), since $\bar{V}=\mu \bar{B}$ and $\bar{B} \cdot \nabla(\bar{J}-\mu \bar{\omega})=0$ in $D$.

We obtain the following expression for the total pressure head $P=p+\frac{1}{2} V^{2}$ :

$$
P(\bar{\psi})=\int_{0}^{\bar{\psi}} \lambda(\sigma) d \sigma+\beta^{-1} s(\bar{\psi})+\text { const }
$$

where $s(\bar{\psi})$ is the equilibrium entropy density

$$
s(\bar{\psi}(x))=-\int_{R^{4}} \rho_{. x}(y) \log \rho_{. x}(y) d y=2+\log \frac{4 \pi^{2}}{\beta^{2}\left[1-\mu(\bar{\psi}(x))^{2}\right]}
$$

We verify this equilibrium relation by referring to the steady version of (2.2), from which we recognize that $\Lambda(\bar{\psi})=d P / d \bar{\psi}$. The equivalence of (3.24) with the definition of $A$ in (3.23) then follows immediately. Accordingly, we see that the profile function $\lambda(\bar{\psi})$ represents the contribution to the pressure gradient from the mean field, while $\beta^{-1} d s / d \bar{\psi}$ represents the contribution from the fluctuations. 


\subsection{Predictions of the Continuum Model}

In general, there is a nontrivial coupling between the equation for the mean fieid-flow and the probability distribution for the local fluctuations in the statistical equilibrium problem. Indeed, the equilibrium equation for the mean flux function $\bar{\psi}$ contains the inverse temperature $\beta$ and the correlation $\mu$, while the energy and generalized cross-helicity constraints, which determine those parameters, also involve $\bar{\psi}$. For this reason, we cannot expect to solve the mean field-flow equations independently of the global constraints on $E, F_{i}$, and $K_{i}$, even though these equations are identical with the steady ideal MHD equations. Rather, the profile functions occurring in those equations, which are arbitrary from a deterministic standpoint, are required to satisfy the family of constraints in the maximum entropy principle. A complete solution of this problem, therefore, appears to require a numerical method of solutions-say, an iterative procedure that alternates between solves in $\bar{\psi}, \alpha_{1}, \ldots, \alpha_{1}$ and solves in $\beta, \gamma_{1}, \ldots, \gamma_{h}$. We shall not attempt to develop such a method in the present paper. Instead, we shall be content to describe some of the general qualitative features of the constrained solutions.

The simplest case to analyze is the magnetostatic case, in which the mean velocity field vanishes $(\bar{V}=0)$. By virtue of $(3.13)$, this case corresponds to the vanishing of the correlation profile function $\mu$, which holds whenever the cross-helicity constraint values $K_{j}^{0}$ are taken to be zero, as is evident from (3.21). From (3.12) we obtain the following equation for the mean flux function $\bar{\psi}$ :

$$
-\Delta \bar{\psi}=\lambda(\bar{\psi})
$$

where the profile function $\lambda$ is given by (3.10). Equation (3.26) is the wellknown Grad-Shafranov equation from plasma physics, ${ }^{(4.24)}$ in which $\lambda=d p / d \bar{\psi}$ defines the pressure profile. It is identical to the variational equation for the following multiconstrained minimization problem:

$$
E(\bar{\psi})=\frac{1}{2} \int_{D}|\operatorname{Curl} \bar{\psi}|^{2} d x \rightarrow \min \quad \text { over } \quad F_{i}(\bar{\psi})=F_{i}^{0} \quad(i=1, \ldots, h)
$$

in which $\lambda(\bar{\psi})=\sum_{i} \lambda_{i} f_{i}^{\prime}(\bar{\psi})$ is determined by the multipliers $\lambda_{i}$ for the flux constraints. The mean-field problem can therefore be viewed as a nonlinear elliptic eigenvalue problem with many parameters $\lambda_{i}$. For each admissible vector of constraint values $F^{0}=\left(F_{1}^{0}, \ldots, F_{h}^{0}\right)$, a solution pair $(\bar{\psi}, \lambda)$ exists and is regular. ${ }^{(2)}$ The uniqueness of this solution is not assured in general, however, since the solution branches can bifurcate. Nevertheless, robust numerical algorithms are available to solve variational problems of this 
kind. ${ }^{(12.38)}$ Accordingly, we will consider the mean field $\bar{B}$ as essentially determined by the given constraint values of flux functionals $F_{i}$, leaving aside the precise description of solution branches.

The variance of each component of the field and the flow in the magnetostatic case is equal to $\beta^{-1}$, where $\beta$ is the energy multiplier or inverse absolute temperature. All of these components are uncorrelated, as is immediate from (3.16). Equation (3.20) for the total energy simplifies to

$$
E^{0}=E(\bar{\psi})+2 \beta^{-1}|D|
$$

Thus, the temperature $\beta^{-1}$ is determined uniquely by the constraint value for the energy functional $E$. Now we see that the statistical equilibrium state with vanishing cross-helicity is completely specified whenever $E^{0} \geqslant E(\bar{\psi})$.

When we split the energy into its magnetic and kinetic parts, we find that $E_{\text {nag }}=E(\bar{\psi})+\beta^{-1}|D|$ and $E_{\text {kin }}=\beta^{-1}|D|$. It follows that the difference between the total energy $E^{0}$ and the energy of the coherent structure $E(\bar{\psi})$ resides in the local fluctuations, where it is equipartitioned into magnetic and kinetic parts equal to $\beta^{-1}|D|$. This result is clearly in good agreement with the picture of ideal turbulence as a sea of high-wavenumber random Alfven waves. ${ }^{(4)}$ A noteworthy consequence of this result is the prediction that the equilibrium value of the ratio $E_{\text {mag }} / E_{\text {kin }}$ is always greater than one. This prediction of our continuum model is in excellent agreement with the results of direct numerical simulations, ${ }^{(5-7)}$ which provide convincing evidence for the relaxation of this ratio to a final value near unity, even for initial values as small as $1 / 25$. Figure 1 shows one such result taken from ref. 6.

For these magnetostatic solutions, we observe a qualitative difference between the high-energy regime $\left[E^{0} \gg E(\bar{\psi})\right]$ and the low-energy regime $\left[E^{0} \approx E(\bar{\psi})\right]$. On the one hand, as $E^{0} \rightarrow+\infty$, we find that $\beta \rightarrow 0$, and hence that the variance of $B$ and $V$ diverges. The equilibrium state therefore resembles homogeneous turbulence: the variance of its local fluctuations is large and constant over the domain $D$, while its mean field is bounded. The coherent structure is thus obliterated by the fluctuations. On the other hand, as $E^{0} \rightarrow E(\bar{\psi})$, we have that $\beta \rightarrow+\infty$, and hence that the variance of $B$ and $V$ tends to zero. This means that the field-flow state relaxes into a nonturbulent magnetostatic equilibrium, in which the fluctuations about the coherent mean field are negligible. These features of the typical final state agree well with the results of direct numerical simulations with small $\mu .^{(5-7.21)}$

The effects of $B-V$ correlations are readily examined by considering a simplified version of the maximum entropy problem in which the classical 


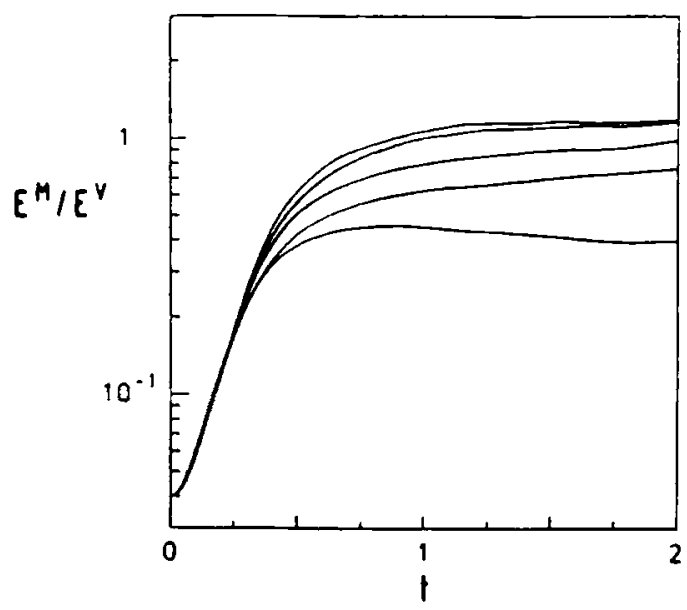

Fig. 1. Relaxation of the ratio of magnetic energy $E^{\prime \prime}$ to kinetic energy $E^{\prime}$, as demonstrated by the direct numerical simulations of Biskamp and Welter ${ }^{(6)}$ of slightly dissipative 2D MHD in a periodic box. The different curves correspond to simulations with identical initial conditions and different levels of dissipation. With increasing Reynolds number, $E^{\prime \prime} / E^{\prime \prime}$ appears to converge to a value between 1 and 2 .

quadratic cross-helicity constraint $K^{0}=\int \overline{B \cdot V} d x$ is imposed, rather than the full family of generalized cross-helicities. As this simplified version of the statistical equilibrium problem is analyzed in detail in a companion paper, ${ }^{(19)}$ we content ourselves here with a brief discussion of its main predictions. Most importantly, the magnetic-velocity correlation defined in (3.17) becomes the constant $\mu=-\gamma^{\prime} / \beta$, where $\gamma$ is the multiplier for the quadratic cross-helicity constraint. As a result, the equilibrium probability density $\rho_{x}(y)$ has a constant covariance matrix. Furthermore, the mean field-flow Eqs. (3.12)-(3.13) simplify when $\mu$ is constant, in the same manner as in the magnetostatic case. Again the mean magnetic field is a critical point of the magnetic energy functional $E(\bar{\psi})$, subject to the given flux constraints. The energy and cross-helicity constraints take the simple forms

$$
\begin{aligned}
& E^{0}=\left(1+\mu^{2}\right) E(\bar{\psi})+\frac{2|D|}{\beta\left(1-\mu^{2}\right)} \\
& K^{0}=2 \mu E(\bar{\psi})+\frac{2 \mu|D|}{\beta\left(1-\mu^{2}\right)}
\end{aligned}
$$

We note that $\mu$ has the same sign as $K^{0}$, and that $\mu$ tends to zero as $K^{0} \rightarrow 0$. The entropy of the statistical equilibrium density $\rho$ is found to be

$$
S(\rho)=|D| \log \left\{\left[E^{0}-\left(1+\mu^{2}\right) E(\bar{\psi})\right]^{2}-\left[K^{0}-2 \mu E(\bar{\psi})\right]^{2}\right\}+\text { const }
$$


where the constant depends only on $|D|$. This expression is obtained by substituting (3.29) and (3.30) into the general formula (3.25). From it we draw the interesting conclusion that the relaxed state $\rho$ balances two competing tendencies: the one, to maximize the fluctuation part of the energy; the other, to minimize the fluctuation part of the cross-helicity. Since the mean field $\bar{B}$ is fixed by the flux constraints, this balance is achieved by the correlation $\mu$, which is the only free parameter in the entropy expression (3.31). Hence, we find that $\mu$ is the solution of the associated critical point equation

$$
E(\bar{\psi}) \mu^{3}-\left(E(\bar{\psi})+E^{0}\right) \mu+K^{0}=0
$$

Under some restrictions on $E^{\prime \prime}$ and $K^{0}$, this equation can be shown to admit a unique solution $\mu \in(-1,1)$. Once $\mu$ is known, $\beta$ is found from either (3.29) or (3.30), and the statistical equilibrium state $\rho$ is thus completely determined. The details are presented in ref. 19 .

Of particular interest is the regime in which $B$ and $V$ are strongly correlated. Provided that $E^{0}>2 E(\bar{\psi})$, the above analysis shows that the correlation $\mu$ approaches 1 , and the variance $\beta^{-1}\left(1-\mu^{2}\right)^{-1}$ approaches $E^{0}-2 E(\bar{\psi})$, as the prescibed ratio $K^{0} / E^{0}$ goes to 1 , its largest possible value. Consequently, the field and the flow become completely correlated in this limit, and the marginal distributions of $B(x)$ and $V(x)$ converge to the same Gaussian distribution with mean $\bar{B}$ and componentwise variance $E^{0}-2 E(\bar{\psi})$. In short, the field and the flow become statistically indistinguishable in this degenerate limit. This vivid effect is confirmed by direct numerical simulations, ${ }^{(36)}$ which show that the field and flow tend to align dynamically when the initial ratio of quadratic cross-helicity to energy is taken above a certain threshold value. Similarly, as $K^{0} / E^{0}$ goes to -1 , its smallest possible value, the correlation $\mu$ tends to -1 , and the field and the flow become anti-aligned.

In the general case in which the profile function $\mu(\sigma)$ is not constant, the properties of the constrained solutions are difficult to extract from the statistical equilibrium equations. Nevertheless, we can infer the following qualitative features. First, we note that in all regimes the equilibrium ratio $E_{\text {mag }} / E_{\text {kin }}$ is greater than one, regardless of its initial value. The model therefore captures the amplification and saturation of the magnetic field observed in turbulence simulations when the initial ratio is small. ${ }^{(6)}$ Second, we see that the relationship $\bar{V}=\mu \bar{B}$ is always predicted, and that the scalar factor between the mean field and flow is identified with the correlation $\mu(\bar{\psi})$ for their fluctuations. Third, we deduce from (3.15) and (3.16) that large fluctuations of both $B$ and $V$ are expected wherever this correlation is strong. This prediction is especially interesting when $\mu(\sigma)$ is 
nonconstant, since then the variance of the fluctuations is enlarged near those flux surfaces where $\mu(\bar{\psi})^{2} \approx 1$. Furthermore, the current density is predicted to peak at these flux surfaces, owing to the presence of the second term in the profile function $A$ exhibited in (3.23). This behavior is in accord with the observed behavior of turbulent states, which show the concentration of fluctuations around definite magnetic surfaces usually connected with X-points. ${ }^{15.31)}$ The analysis of this effect, however, requires that we determine variability of the equilibrium profile function $\mu(\sigma)$ in the constrained solutions. We defer such an analysis to subsequent work.

\section{JUSTIFICATION OF THE CONTINUUM MODEL}

\subsection{Discrete Model}

The formulation of the continuum model in Section 2 relies on two postulates: the ergodic hypothesis and a separation-of-scales hypothesis. Under these hypotheses we are able to describe the typical state of turbulence macroscopically by the $x$-parametrized probability density $\rho_{x}(y)$. Our derivation of the maximum entropy principle (2.24) that determines the most probable macrostate $\rho_{x}(y)$, however, is heuristic rather than rigorous. In three particular respects the formulation requires further justification: (1) the neglect of correlations between points, which allows us to use the single-point distributions; (2) the specific form of the entropy functional, which is based on an a priori distribution that is uniform on $D \times R^{4} ;(3)$ the vanishing of the variance of $\psi$, which permits us to express magnetic surface quantities in terms of the mean flux function $\bar{\psi}$. In this section, we therefore develop a lattice model of ideal magnetofluid turbulence, which tends in the limit to the continuum model. In the course of this development, we justify the continuum model rigorously and we connect it to some standard concepts in statistical mechanics ${ }^{(1,2.11)}$ and fluid turbulence. ${ }^{(10,22)}$

Throughout this section we restrict our attention to a doubly periodic spatial geometry. These familiar boundary conditions allow us to utilize the relationship between the discretization of $x$-space and the truncation of $k$-space in a simple and concrete way. We let

$$
D=\left\{x=\left(x_{1}, x_{2}\right) \in R^{2}:\left|x_{1}\right|<L_{1} / 2,\left|x_{2}\right|<L_{2} / 2\right\}
$$

be the fundamental period domain, and we normalize its area $|D|=$ $L_{1} L_{2}=1$. For large, even integers $N_{1}$ and $N_{2}$, we introduce the discrete domain $D_{N}$ consisting of equally spaced nodes $x_{n}=\left(n_{1} \Delta x_{1}, n_{2} \Delta x_{2}\right)$ with $\Delta x_{1}=L_{1} / N_{1}$ and $\Delta x_{2}=L_{2} / N_{2}$, where the multi-index $n=\left(n_{1}, n_{2}\right)$ runs 
over the integral lattice with $-N_{1} / 2<n_{1} \leqslant N_{1} / 2,-N_{2} / 2<n_{2} \leqslant N_{2} / 2$. The discrete grid $D_{N}$ then contains $N:=N_{1} N_{2}$ nodes.

Any microstate $Y(x)$ defined for $x \in D$ can be sampled at the nodes $x_{n} \in D_{N}$ to produce the discretized state vector $Y=\left(Y_{n}\right)$ whose components represent the nodal values $Y\left(x_{n}\right)$. The discretized phase space is therefore $R^{4 N}$. The Euclidean inner product on this space will be denoted by

$$
(Y, \tilde{Y})_{N}:=\frac{1}{N} \sum_{n} Y_{n} \cdot \widetilde{Y}_{n}
$$

where the factor $1 / N$ is included to maintain contact with the continuum limit. The Euclidean norm will be written as $\|Y\|_{N}^{2}=(Y, Y)_{N}$. Here and throughout the sequel, sums over $n$ are understood to extend over the $N$ nodes in $D_{N}$.

The discrete Fourier transform of a microstate $Y$ on $D_{N}$ involves the $N$ wavevectors $k_{m}=\left(m_{1} \Delta k_{1}, m_{2} \Delta k_{2}\right)$ with $\Delta k_{1}=2 \pi / L_{1}$ and $\Delta k_{2}=2 \pi / L_{2}$, where $m=\left(m_{1}, m_{2}\right)$ is a multi-index running over $-N_{1} / 2<m_{1} \leqslant N_{1} / 2$, $-N_{2} / 2<m_{2} \leqslant N_{2} / 2$. The inversion formula for the transform reads

$$
Y_{n}=\sum_{k} \hat{Y}_{k} e^{i k \cdot x_{n}} \quad \text { with } \quad \hat{Y}_{k}:=\frac{1}{N} \sum_{n} Y_{n} e^{-i k \cdot x_{n}}
$$

Here and henceforth sums over $k$ extend over the $N$ wavevectors $k=k_{m}$. This formula establishes a linear, one-to-one correspondence between the real state vectors $Y$ and those complex amplitude vectors $\hat{Y}$ which satisfy the compatibility conditions $\hat{Y}_{-k}=\hat{Y}_{k}^{*}$. The Parseval identity for $(4.1)$ is

$$
\frac{1}{N} \sum_{n}\left|Y_{n}\right|^{2}=\sum_{k}\left|\hat{Y}_{k}\right|^{2}
$$

in which $\left|\hat{Y}_{k}\right|^{2}=\hat{Y}_{k} \cdot \hat{Y}_{k}^{*}$ uses the Hermitian dot product. By virtue of this identity, the discrete Fourier transform constitutes an isometry between Euclidean space $R^{4 N}$ and the subspace of the Hermitian space $C^{4 N}$ defined by the compatibility conditions. We refer the reader to standard texts ${ }^{(35.9)}$ for detailed treatments.

While the doubly periodic boundary conditions are natural and convenient in formulating a discrete model, they suffer from the minor drawback that they permit arbitrary $x$-translations. Rather than including additional conserved quantities as constraints in the statistical equilibrium model, we shall eliminate these $x$-translations by imposing some finite symmetry conditions on the microstates. These conditions amount to posing 
the problem on the quarter domain $D^{+}=D \cap\left\{x_{1}, x_{2}>0\right\}$ with ideal boundary conditions on $\partial D^{+}$. The continuous microstate $Y=(\operatorname{Curl} \psi$, Curl $\phi$ ) then possesses the symmetries in $x_{1}$ and $x_{2}$ inherited from those of $\psi$ and $\phi$, on which we impose $\psi=0=\phi$ on $\partial D^{+}$, and which we extend to $D$ by odd reflection in $x_{1}$ and $x_{2}$. In other words, we imagine the physical domain to be $D^{+}$and assume that $\partial D^{+}$is a perfectly conducting and slipping boundary. The Fourier representations of $\psi$ and $\phi$ then become sine expansions. The components of $Y$ are similarly extended to $D$ by reflection (even or odd, depending on the component and the variable), and so are represented by reduced expansions (cosine or sine expansions). In what follows we shall enforce these symmetry conditions on the continuous and discrete versions of the problem. We note specifically that the $k=0$ Fourier amplitudes vanish.

We can take the discretized microscopic dynamics to be governed by the truncated spectral form of the primitive equations. This form, which is often described in the literature, defines the evolution of the complex amplitudes $\hat{Y}_{k}(t)$. The detailed equations for $\hat{Y}_{k}$ are not needed for our purposes. We use only the Liouville property satisfied by the truncated $k$-space equations: the phase flow conserves (Hermitian) phase volume. This crucial property is demonstrated by several authors. ${ }^{(22,23)}$ By invoking the inverse discrete Fourier transform we can map this dynamics from the truncated $k$-space to the discrete $x$-space. In this way we can define the microscopic dynamics in the discretized primitive variables $Y_{n}$ over $D_{N}$. Since the mapping between $\hat{Y}$ and $Y$ is a linear isometry, we immediately infer that the induced phase flow on the $x$-space $R^{4 N}$ is volume-preserving. In other words, our discrete $x$-space dynamics satisfies the Liouville property.

We now approximate the conserved quantities for the continuum equations by their discrete analogues, namely,

$$
\begin{aligned}
& E=\frac{1}{2 N} \sum_{n}\left|B_{n}\right|^{2}+\left|V_{n}\right|^{2}=\frac{1}{2} \sum_{k}\left|\hat{B}_{k}\right|^{2}+\left|\hat{V}_{k}\right|^{2} \\
& F_{i}=\frac{1}{N} \sum_{n} f_{i}\left(\psi_{n}\right) \\
& K_{i}=\frac{1}{N} \sum_{n} B_{n} \cdot V_{n} f_{i}^{\prime}\left(\psi_{n}\right)
\end{aligned}
$$

The energy $E$ is conserved exactly by the discretized dynamics, as an analysis of the detailed equations shows. The generalized fluxes $F_{i}$ and cross-helicities $K_{i}$, however, are conserved only in the limit as $N \rightarrow \infty$, unless very particular basis functions are used $\left[f(\psi)=\psi^{2} / 2\right.$ for $F$ and 
$f(\psi)=\psi$ for $K$ ]. Even though the nonlinear and nonlocal dependence of these quantities on $\psi$ destroys their exact conservation, we shall adopt these approximate dynamical invariants as the constraints for our lattice model.

We note that the flux function used in (4.4) and (4.5) is defined by the $k$-space relation

$$
\hat{\psi}_{k}=i|k|^{-2} k \times \hat{B}_{k}
$$

Together with the discrete Fourier transform, this relation provides the discretized form of the operator $\mathrm{Curl}^{-1}$. We shall denote this $x$-space operator by $\mathscr{A}$ and its adjoint with respect to $(\cdot, \cdot)_{N}$ by $\mathscr{A}^{\dagger}$.

\subsection{Separation of Scales}

With a discrete microscopic model in hand, we now consider a macroscopic description in terms of a probability distribution $p^{N}(d Y)$ on the phase space $R^{4 N}$. For the sake of definiteness, we write $p^{N}(d Y)=\rho^{N}(Y) d Y$ and take the macrostate to be the probability density $\rho^{N}(Y)$ with respect to $4 N$-volume $d Y$. Any functional $A(Y)$ on the discrete phase space then defines a random variable whose expectation (mean) is denoted by

$$
\langle A\rangle=\int_{R^{+N}} A(Y) \rho^{N}(Y) d Y
$$

We seek a probability density that describes the statistical equilibrium state for the discrete dynamics. In accord with the standard ergodic hypothesis, we define this state to be the density $\rho^{N}$ that maximizes the entropy

$$
S\left(\rho^{N}\right)=\frac{-1}{N} \int_{R^{* N}} \rho^{N}(Y) \log \rho^{N}(Y) d Y
$$

subject to the constraints associated with the conserved quantities $E, F_{i}, K_{i},{ }^{11.2 .181}$ The form of this entropy functional is dictated by the Liouville property for the discrete dynamics, which demands that the density $\rho^{N}(Y)$ be relative to the invariant measure $d Y$. Thus, the objective functional in our maximum entropy principle is uniquely determined up to a multiplicative factor, which we take to be $1 / N$ in order to have a finite entropy in the continuum limit.

Unlike the usual canonical ensemble, however, the constraint functionals for flux and cross-helicity are not simply taken to be the expectations $\left\langle F_{i}\right\rangle$ and $\left\langle K_{i}\right\rangle$ of the functionals in (4.4) and (4.5). This unorthodox aspect of our model is dictated by the particular form of the dynamical 
constraints for ideal turbulence. In contrast to the energy functional $E$, which is simply quadratic in $B$ and $V$, the functionals $F_{i}$ and $K_{i}$ involve the flux function $\psi$, which has distinctly different statistical behavior from that of $B$ and $V$. Consequently, the usual canonical measure formed from a linear combination of $E, F_{i}$, and $K_{i}$ can fail to provide a meaningful continuum limit as $N \rightarrow \infty$.

Before giving our formulation, therefore, let us briefly review the theory of absolute equilibrium distributions, ${ }^{(13,22)}$ in order to explain the role of the additional dynamical constraints in the Gibbs measure. This theory is derived from the usual canonical ensemble based on energy $E$, quadratic flux $F$ [with $f(\psi)=\psi^{2} / 2$ ] and classical cross-helicity $K$ [with $f(\psi)=\psi$ ]. A straightforward computation yields the following results. ${ }^{(13.22 .34)}$ First, these statistical equilibrium distributions have zero mean: $\left\langle\hat{Y}_{k}\right\rangle=0$ for all $k$. Second, they predict that the given energy $E^{\prime \prime}$ splits into a part $\theta E^{0}$ that equipartitions among all the modes $k_{\min }<|k| \leqslant k_{\max }$ and a part $(1-\theta) E^{\prime \prime}$ that resides in fluctuations at $|k|=k_{\min }$. From these properties, we see that the theory fails to predict a nontrivial coherent structure in the mean. Instead, it puts some energy into a fluctuating eigenmode at the lowest wavenumber. While the equipartition of energy among the high wavenumbers is consistent with the ideal model, the presence of finite fluctuations at the lowest wavenumber violates the principle on which the canonical ensemble rests, since the fluctuations of the energy about its prescribed mean value do not tend to zero as $k_{\max } \rightarrow \infty$. In other words, the equivalence of ensembles breaks down. Moreover, the prediction of a zero mean state is inconsistent with the microcanonical ensemble when $E^{(0)}$ is close to the minimum energy subject to given values of $F^{\prime \prime}$ and $K^{\prime \prime}$. In such cases the microscopic constraint surface becomes disconnected into two components distinguished by the sign of the eigenmode at $k=k_{\min }$. The microcanonical ensemble for one fixed component then produces a nonzero mean state with small fluctuations in every mode. The usual canonical ensemble, however, predicts finite fluctuations around the zero state in the lowest mode.

These considerations motivate our particular formulation of the constraints on flux and cross-helicity in the maximum entropy principle. We base our model on the separation-of-scales hypothesis, which we now articulate precisely. In our discrete model, we separate the modal energy density $\frac{1}{2}\left\langle\left|\hat{Y}_{k}\right|^{2}\right\rangle$ into mean and fluctuation parts, which we require to satisfy

$$
\begin{aligned}
\frac{1}{2}\left|\left\langle\hat{Y}_{k}\right\rangle\right|^{2} \leqslant \varepsilon_{k} \quad \text { where } \quad \sum_{k} \varepsilon_{k} \leqslant E^{0} \\
\frac{1}{2}\left\langle\left|\hat{Y}_{k}-\left\langle\hat{Y}_{k}\right\rangle\right|^{2}\right\rangle \leqslant C E^{\prime \prime} / N
\end{aligned}
$$


for a sequence of constants $\varepsilon_{k}$ and a constant $C$, both independent of $N$ as $N \rightarrow \infty$. These conditions imply that the given energy $E^{0}=\langle E(Y)\rangle$ resides partly in a uniformly square-integrable mean state $\langle Y\rangle$ and partly in fluctuations, where it is partitioned uniformly (up to a constant factor) among all the $N$ modes. For large $N$, therefore, we see that the separation of scales imposed in (4.8)-(4.9) causes the mean to concentrate in the low wavenumbers and the fluctuations to spread out to the high wavenumbers. We emphasize that these conditions constitute a hypothesis used to derive the model, not a conclusion deduced from it. Nevertheless, this single hypothesis is the only special ingredient needed to arrive at our model. In what follows we denote fluctuations by a prime: $Y^{\prime}=Y-\langle Y\rangle$, and so forth.

A crucial consequence of the separation of scales is the asymptotic smallness of the fluctuations of $\psi$. From (4.6), we obtain the estimate

$$
\begin{aligned}
\left\langle\left\|\psi^{\prime}\right\|_{N}^{2}\right\rangle & =\sum_{k}\left\langle\left|\hat{\psi}_{k}^{\prime}\right|^{2}\right\rangle \\
& \leqslant \sum_{k}|k|^{-2}\left\langle\left|\hat{Y}_{k}^{\prime}\right|^{2}\right\rangle \\
& \leqslant C E^{\prime \prime} N^{-1} \sum_{k}|k|^{-2} \\
& =O\left(N^{-1} \log N\right)
\end{aligned}
$$

recalling that the sums are evaluated over the two-dimensional lattice of wavevectors $k=k_{m}$. We conclude that in a strong sense the fluctuations of $\psi$ are negligible for large $N$. Of course, an analogous statement holds for $\phi$.

We can now infer the correct form of the constraints on $F_{i}$ and $K_{i}$, by making a formal expansion in $\psi$ around $\langle\psi\rangle$. For the flux constraints we get

$$
\begin{aligned}
\left\langle F_{i}(Y)\right\rangle & =\frac{1}{N} \sum_{n} f_{i}\left(\left\langle\psi_{n}\right\rangle\right)+\frac{1}{2 N} \sum_{n} f_{i}^{\prime \prime}\left(\left\langle\psi_{n}\right\rangle\right)\left\langle\left(\psi_{n}^{\prime}\right)^{2}\right\rangle+\cdots \\
& =F_{i}(\langle Y\rangle)+O\left(N^{-1} \log N\right)
\end{aligned}
$$

assuming that $f_{i}^{\prime \prime}$ is uniformly bounded over the range of the mean flux function. For the cross-helicity constraints, using a similar but more involved analysis, we obtain

$$
\left\langle K_{i}(Y)\right\rangle=\frac{1}{N} \sum_{n}\left\langle B_{n} \cdot V_{n}\right\rangle f_{i}^{\prime}\left(\left\langle\psi_{n}\right\rangle\right)+O\left(N^{-1 / 2}(\log N)^{1 / 2}\right)
$$


In view of these expansions, we formulate our statistical equilibrium model by retaining the leading terms for $\left\langle F_{i}\right\rangle$ and $\left\langle K_{i}\right\rangle$, rather than the full expressions for each of them. This procedure, which we prove in Section 4.4 to be consistent with the desired properties of the ensemble, effectively enforces the separation-of-scales conditions on the solution $\rho^{N}(Y)$ to the maximum entropy problem.

\subsection{Implicit Canonical Ensemble}

We define a statistical equilibrium macrostate $\rho^{N}(Y)$ to be a solution to the multiconstrained optimization problem

$$
\begin{array}{r}
S\left(\rho^{N}\right) \rightarrow \max \quad \text { subject to: } \\
\frac{1}{2 N} \sum_{n}\left\langle\left|B_{n}\right|^{2}+\left|V_{n}\right|^{2}\right\rangle=E^{\prime \prime} \\
\frac{1}{N} \sum_{n} f_{i}\left(\left\langle\psi_{n}\right\rangle\right)=F_{i}^{0} \\
\frac{1}{N} \sum_{n}\left\langle B_{n} \cdot V_{n}\right\rangle f_{i}^{\prime}\left(\left\langle\psi_{n}\right\rangle\right)=K_{i}^{0}
\end{array}
$$

Unlike the usual maximum entropy principle, the density $\rho^{N}$ enters into the constraints (4.14) and (4.15) nonlinearly. Consequently, we refer to the solution as the "implicit canonical measure," emphasizing that it is determined by solving the statistical equilibrium equations implicitly in $\rho^{N}$. This implicit dependence acts through the mean flux function $\langle\psi\rangle=\mathscr{A}\langle B\rangle$. with the result that the nonlinearity occurs in the mean-field equations. Accordingly, this implicit canonical measure defines a certain "mean-field theory" for the Gibbs measure associated with ideal magnetofluid turbulence. The purely quadratic dependence of the constraints (4.13) and (4.15) on the primitive variables $B$ and $V$ results in Gaussian distributions without spatial correlations. This property, which reflects the separation-ofscales hypothesis, makes the problem tractable.

The variational equations for this discrete model parallel those for the continuum model (Section 3.1), with the difference that $p^{N}(Y)$ is now a joint probability density on the phase space $R^{4 N}$. In terms of the discrete inner product $(\cdot, \cdot)_{N}$ and norm $\|\cdot\|_{N}$ and the linear operators $\mathscr{A}$ and $\mathscr{A}^{\dagger}$, which are the discretizations of $\mathrm{Curl}^{-1}$ and $\mathrm{curl}^{-1}$, we find that 


$$
\begin{aligned}
& \delta S=\frac{-1}{N} \int \log \rho^{N} \delta \rho^{N} d Y \\
& \delta E=\int \frac{1}{2}\left(\|B\|_{N}^{2}+\|V\|_{N}^{2}\right) \delta \rho^{N} d Y \\
& \delta F_{i}=\int\left(B, \mathscr{A}^{\dagger} f_{i}^{\prime \prime}(\langle\psi\rangle)\right)_{N} \delta \rho^{N} d Y \\
& \delta K_{i}=\int\left(B, f_{i}^{\prime}(\langle\psi\rangle) V+\mathscr{A}^{+}\left[\langle B \cdot V\rangle f_{i}^{\prime \prime}(\langle\psi\rangle)\right]\right)_{N} \delta \rho^{N} d Y
\end{aligned}
$$

As in the continuum calculations, we let $\beta, \alpha_{i}, \gamma_{i}$ be the multipliers corresponding to $E, F_{i}, K_{i}$, respectively, and we introduce the profile functions $\lambda(\sigma)$ and $\mu(\sigma)$ defined as in (3.10). We then obtain the (implicit) partition function

$$
Z=\int_{R^{4 . S}} \exp (-\beta N Q(Y)) d Y
$$

where $Q$ is the quadratic form in $B_{n}, V_{n}$ given by

$$
Q=\frac{1}{2}\|B\|_{N}^{2}+\frac{1}{2}\|V\|_{N}^{2}-\left(B, \mathscr{A}^{\dagger} \lambda\right)_{N}-\left(B, \mu V+\mathscr{A}^{\dagger}\left[\langle B \cdot V\rangle \mu^{\prime}\right]\right)_{N}
$$

Here, $\lambda_{n}=\lambda\left(\left\langle\psi_{n}\right\rangle\right)$, and similarly for $\mu$. A straightforward calculation yields the implicit canonical density

$$
\begin{aligned}
\rho^{N}(Y) & =\prod_{n} \rho_{n}\left(Y_{n}\right) \\
& =\prod_{n}\left(\frac{\beta}{2 \pi}\right)^{2}\left(1-\mu_{n}^{2}\right) \exp \left[-\frac{\beta}{2}\left(\left|B_{n}^{\prime}\right|^{2}+\left|V_{n}^{\prime}\right|^{2}-2 \mu_{n} B_{n}^{\prime} \cdot V_{n}^{\prime}\right)\right]
\end{aligned}
$$

with mean field $\langle B\rangle$ and mean flow $\langle V\rangle$ satisfying

$$
\begin{aligned}
& \langle B\rangle=\mathscr{A}^{\dagger}\left(\lambda+\langle B \cdot V\rangle \mu^{\prime}\right)+\mu\langle V\rangle \\
& \langle V\rangle=\mu\langle B\rangle
\end{aligned}
$$

(a prime denotes $d / d \sigma$, when applied to a basis function $f_{i}$ or a profile function $\lambda$ or $\mu$ ).

The decisive result of these calculations is the statistical independence of the Gaussian fluctuations $Y_{n}^{\prime}$ at each node $x_{n} \in D_{N}$. This property of the maximum entropy solution $\rho^{N}$ is a direct consequence of the particular 
form of the constraints (4.13)-(4.15). We see therefore that the desired separation of scales follows from these implicit dynamical constraints. The nodal densities $\rho_{n}\left(Y_{n}\right)$ are clearly a discretization of the local probability densities $\rho_{x}(y)$ in the continuum model.

The analysis now follows exactly as in the continuous model. Equations (4.23) and (4.24), being the analogues of (3.12) and (3.13), are reducible to a mean-field equation that is the discrete version of (3.22). This equation determines $\langle B\rangle$ (or equivalently $\langle\psi\rangle$ ) implicitly in terms of the profile functions $\lambda(\sigma)$ and $\mu(\sigma)$, which in turn are determined by the constraints. In equilibrium, the constraints for the discrete model are

$$
\begin{aligned}
E(\langle B\rangle,\langle V\rangle)+\frac{2}{\beta N} \sum_{n} \frac{1}{1-\mu_{n}^{2}} & =E^{0} \\
F_{i}(\langle B\rangle) & =F_{i}^{0} \\
K_{i}(\langle B\rangle,\langle V\rangle)+\frac{2}{\beta N} \sum_{n} \frac{\mu_{n}}{1-\mu_{n}^{2}} f_{i}^{\prime}\left(\left\langle\psi_{n}\right\rangle\right) & =K_{i}^{0}
\end{aligned}
$$

using an analysis as in Section 3.1. The further comments made in Section 3 about how to solve the statistical equilibrium problem carry over to this discrete case without any substantive changes.

\subsection{Concentration Property}

We now complete the justification of our continuum model by showing that as $N \rightarrow \infty$ the implicit canonical density $\rho^{N}$ concentrates around the manifold defined by the microscopic constraints: $E(Y)=E^{\prime \prime}, F_{i}(Y)=F_{i}^{\prime \prime}$, $K_{i}(Y)=K_{i}^{\prime \prime}$. In other words, we establish an equivalence between the microcanonical ensemble and our implicit canonical ensemble. This rigorous demonstration justifies the formal procedure used in Section 4.4 to construct the implicit canonical measure.

Our proof of the concentration result assumes that the $\left(B_{n}, V_{n}\right)$ distributions are nondegenerate, in the sense that the correlation $\mu_{n}$ is bounded away from \pm 1 uniformly over $x_{n} \in D_{N}$ as $N \rightarrow \infty$. In the discussion that follows, therefore, we impose the condition that $\max _{n}\left|\mu_{n}\right| \leqslant \mu_{*}<1$ for all $N$. This limit condition for the discrete model amounts to a regularity condition on the solution to the continuum model, as is evident from the discussion in Section 3. We therefore adopt the view that it can be verified a posteriori. Under this condition on the $B-V$ correlation, the mean fieldflow $\left\langle Y_{n}\right\rangle$ and the variance $\left(\left|Y_{n}^{\prime}\right|^{2}\right\rangle$ remain uniformly bounded over $D_{N}$ as $N \rightarrow \infty$. Moreover, since the distributions of the $Y_{n}$ are Gaussian, all higher moments are similarly bounded in terms of the mean and variance. 
We begin our analysis by showing that the variance of $\psi_{n}$ for each $n$ tends to zero as $N \rightarrow \infty$. This property, which constitutes the discrete version of (2.19), is fundamental to our theory. We first express the flux function as a convolution over $D_{N}$,

$$
\psi_{n}=\frac{1}{N} \sum_{v} \Gamma^{N}\left(x_{n}-x_{v}\right) \times B_{v}
$$

using the Green function

$$
\Gamma^{N}\left(x_{n}\right)=\sum_{k} i|k|^{-2} k e^{i k \cdot x_{n}}
$$

This formula follows easily from (4.6). Next, we calculate the variance of each component of $B_{n}$ to be $\beta^{-1}\left(1-\mu_{n}^{2}\right)^{-1}$, using the discrete analogue of (3.15). Since the $B_{n}$ are statistically independent, we then conclude that

$$
\begin{aligned}
\operatorname{var} \psi_{n} & \leqslant \frac{1}{\beta\left(1-\mu_{*}^{2}\right) N^{2}} \sum_{1}\left|\Gamma^{N}\left(x_{n}-x_{1}\right)\right|^{2} \\
& =\frac{1}{\beta\left(1-\mu_{*}^{2}\right) N} \sum_{k}\left|\hat{\Gamma}_{k}^{N}\right|^{2} \\
& =O\left(N^{-1} \log N\right)
\end{aligned}
$$

where we used the fact that $\left|\hat{\Gamma}_{k}^{N}\right|=O\left(|k|^{-1}\right)$.

We claim that the statistical equilibrium density $\rho^{N}$ satisfies

$$
\left\langle\left[E(Y)-E^{0}\right]^{2}\right\rangle,\left\langle\left[F_{i}(Y)-F_{i}^{0}\right]^{2}\right\rangle,\left\langle\left[K_{i}(Y)-K_{i}^{0}\right]^{2}\right\rangle \rightarrow 0
$$

as $N \rightarrow \infty$. This property clearly means that the implicit canonical measure is a consistent approximation to the statistical behavior of the underlying microscopic dynamics, assuming the ergodicity of these dynamics over the microcanonical manifold. We proceed to give the proof of each of these three limits.

The energy functional is treated in a routine fashion, since $Y=\left(Y_{n}\right)$ consists of independent Gaussian random vectors. Namely,

$$
\begin{aligned}
\left\langle\left[E(\hat{Y})-E^{0}\right]^{2}\right\rangle & =\left\langle E(Y)^{2}\right\rangle-\langle E(Y)\rangle^{2} \\
& =\frac{1}{4 N^{2}} \sum_{n} \sum_{v}\left\langle\left|Y_{n}\right|^{2}\left|Y_{n}\right|^{2}\right\rangle-\left\langle\left|Y_{n}\right|^{2}\right\rangle\left\langle\left|Y_{n}\right|^{2}\right\rangle \\
& =\frac{1}{4 N^{2}} \sum_{n}\left\langle\left|Y_{n}\right|^{4}\right\rangle-\left\langle\left|Y_{n}\right|^{2}\right\rangle^{2}=O\left(N^{-1}\right)
\end{aligned}
$$


The generalized flux functional is expanded in $\psi_{n}$ using the estimate (4.28). We assume that the basis functions $f_{i}^{\prime}(\sigma)$ are bounded for all $\sigma \in R$, and we obtain

$$
\begin{aligned}
\left\langle\left[F_{i}(Y)-F_{i}^{0}\right]^{2}\right\rangle & =\left\langle\left[\frac{1}{N} \sum_{n} f_{i}\left(\psi_{n}\right)-f_{i}\left(\left\langle\psi_{n}\right\rangle\right)\right]^{2}\right\rangle \\
& =\left\langle\left[\frac{1}{N} \sum_{n} f_{i}^{\prime}\left(\tilde{\psi}_{n}\right) \psi_{n}^{\prime}\right]^{2}\right\rangle \\
& \leqslant\left\langle\left[\frac{1}{N} \sum_{n} f_{i}^{\prime}\left(\tilde{\psi}_{n}\right)^{2}\right] \max _{n}\left(\psi_{n}^{\prime}\right)^{2}\right\rangle \\
& \leqslant C \max _{n} \operatorname{var} \psi_{n} \\
& =O\left(N^{-1} \log N\right)
\end{aligned}
$$

where $\tilde{\psi}_{n}$ denotes some intermediate value between $\psi_{n}$ and $\left\langle\psi_{n}\right\rangle$.

The generalized cross-helicity functional is analyzed by combining the techniques used in (4.30) and (4.31). First, we write $K_{i}(Y)=G_{i}(Y)+$ $H_{i}(Y)$, where

$$
\begin{aligned}
& G_{i}(Y):=\frac{1}{N} \sum_{n} B_{n} \cdot V_{n} f_{i}^{\prime}\left(\left\langle\psi_{n}\right\rangle\right) \\
& H_{i}(Y):=\frac{1}{N} \sum_{n} B_{n} \cdot V_{n}\left[f_{i}^{\prime}\left(\psi_{n}\right)-f_{i}^{\prime}\left(\left\langle\psi_{n}\right\rangle\right)\right]
\end{aligned}
$$

Then we see that

$$
\begin{aligned}
\left\langle\left[K_{i}(Y)-K_{i}^{0}\right]^{2}\right\rangle & =\left\langle G_{i}^{2}\right\rangle-\left\langle G_{i}\right\rangle^{2}+2\left\{\left\langle G_{i} H_{i}\right\rangle-\left\langle G_{i}\right\rangle\left\langle H_{i}\right\rangle\right\}+\left\langle H_{i}^{2}\right\rangle \\
& \leqslant\left\langle G_{i}^{2}\right\rangle-\left\langle G_{i}\right\rangle^{2}+4\left\langle G_{i}^{2}\right\rangle^{1 / 2}\left\langle H_{i}^{2}\right\rangle^{1: 2}+\left\langle H_{i}^{2}\right\rangle
\end{aligned}
$$

where we note that the constraint is equivalent to $\left\langle G_{i}\right\rangle=K_{i}^{0}$.

It suffices therefore to estimate $\left\langle G_{i}^{2}\right\rangle-\left\langle G_{i}\right\rangle^{2}$ and $\left\langle H_{i}^{2}\right\rangle$, the treatments of which follow (4.30) and (4.31), respectively. First, we have

$$
\begin{aligned}
\left\langle G_{i}^{2}\right\rangle-\left\langle G_{i}\right\rangle^{2} & \leqslant \frac{1}{N^{2}} \sum_{n}\left\{\left\langle\left(B_{n} \cdot V_{n}\right)^{2}\right\rangle-\left\langle B_{n} \cdot V_{n}\right\rangle^{2}\right\} f_{i}^{\prime}\left(\left\langle\psi_{n}\right\rangle\right)^{2} \\
& \leqslant \frac{C}{N^{2}} \sum_{n}\left\langle\left|Y_{n}\right|^{4}\right\rangle+\left\langle\left|Y_{n}\right|^{2}\right\rangle^{2} \\
& =O\left(N^{-1}\right)
\end{aligned}
$$


Second, we assume that the basis functions $f_{i}^{\prime \prime}(\sigma)$ are bounded for all $\sigma \in R$, and we get

$$
\begin{aligned}
\left\langle H_{i}^{2}\right\rangle & =\left\langle\left[\frac{1}{N} \sum_{n} B_{n} \cdot V_{n} f_{i}^{\prime \prime}\left(\tilde{\psi}_{n}\right) \psi_{n}^{\prime}\right]^{2}\right\rangle \\
& \leqslant\left\langle\left[\frac{1}{N} \sum_{n}\left(B_{n} \cdot V_{n}\right)^{2} f_{i}^{\prime \prime}\left(\tilde{\psi}_{n}\right)^{2}\right] \max _{n}\left(\psi_{n}^{\prime}\right)^{2}\right\rangle \\
& \leqslant C\left[\frac{1}{N} \sum_{n}\left\langle\left|Y_{n}\right|^{8}\right\rangle\right]^{1 / 2} \max _{n}\left\langle\left(\psi_{n}^{\prime}\right)^{4}\right\rangle^{1 / 2} \\
& \leqslant C \max _{n} \operatorname{var} \psi_{n}=O\left(N^{-1} \log N\right)
\end{aligned}
$$

Putting these estimates together, we obtain

$$
\left\langle\left[K_{i}(Y)-K_{i}^{0}\right]^{2}\right\rangle=O\left(N^{-1 / 2}(\log N)^{1 / 2}\right)
$$

The conditions we place on the basis functions in this analysis are mild. Indeed, these functions define the flux constraints, which actually apply over the range of the mean flux function $\bar{\psi}$. But this range is fixed $a$ priori by the initial state $Y^{(0)}$ that determines the constraint values. Consequently, we can merely extend the basis functions $f_{i}(\sigma)$ to be defined for all $\sigma \in R$ with the desired growth conditions.

\subsection{Continuum Limit}

We end our analysis of the lattice model by indicating how the statistical equilibrium measures $\rho^{N}=\prod \rho_{n}$ converge to the solution $\rho_{x}(y)$ to the continuous maximum entropy problem (2.24). For each $N$, we define an $x$-parametrized probability density $\sigma_{x}^{N}(y)$ on $y \in R^{4}$ by interpolating the discrete measure $\rho^{N}$ from the lattice model. It suffices to set $\sigma_{x}^{N}(y)=\rho_{n}(y)$ whenever $x$ lies in the $\Delta x_{1} \times \Delta x_{2}$ cell at the node $x_{n}$. This piecewiseconstant macrostate $\sigma_{. r}^{N}(y)$ then has the same entropy as $\rho^{N}$ :

$$
\begin{aligned}
S\left(\sigma^{N}\right) & =-\int_{D} \int_{R^{4}} \sigma_{x}^{N}(y) \log \sigma_{x}^{N}(y) d x d y \\
& =-\frac{1}{N} \sum_{n} \int_{R^{4}} \rho_{n}(y) \log \rho_{n}(y) d y \\
& =-\frac{1}{N} \int_{R^{4 . S}} \rho^{N}(Y) \log \rho^{N}(Y) d Y \\
& =S\left(\rho^{N}\right)
\end{aligned}
$$


By standard probability theory ${ }^{(3)}$ we can ensure that the densities $\sigma_{x}^{N}(y)$ converge in the usual weak sense to a density $\rho_{x}(y)$ along a sequence $N \rightarrow \infty$. The uniform bounds on the (local) moments of all orders of $\sigma^{N}$ guarantee that the limit density $\rho$ satisfies the constraints on energy, crosshelicity, and flux. By virtue of the upper semicontinuity of the entropy functional, we can then infer that $p_{r}(y)$ is the solution of the continuous maximum entropy problem (2.24). Thus, we can demonstrate that $\rho^{N}$ converges in an appropriate weak sense to a solution $\rho$ of the continuum model. We omit the details of this proof.

\section{CONCLUSIONS}

We have formulated, analyzed, and justified a statistical equilibrium model of coherent states in two-dimensional magnetofluid turbulence that respects the complete family of conserved integrals for the ideal dynamics. By requiring a separation of scales between the microscopic fluctuations and the macroscopic mean inherent in the turbulent state, we are able to describe it in terms of the $x$-local probability densities $\rho_{x}(y)$ on the primitive field-flow state $Y(x, t)=(B, V)$. The constrained maximum entropy principle defining our continuum model, which is derived intuitively in Section 2 and substantiated rigorously in Section 4, determines the most probable macrostate $\rho_{x}(y)$. We have found that this statistical equilibrium state is a Gaussian probability density, and that its mean is a steady solution of the ideal MHD equations.

Our statistical equilibrium theory has the virtue that it can be formulated directly as a continuum model in $x$-space. Nonetheless, its full justification requires both a discretization of $x$-space and a dual representation in $k$-space. For this reason, we have constructed a lattice model with $N$ grid nodes, and we have proved that it tends to the continuum model as $N \rightarrow \infty$. With the aid of the discrete Fourier transform, we are able to represent the separation-of-scales hypothesis for the model in a $k$-space form. This hypothesis allows us to deduce the constraints for the maximum entropy principle that defines our lattice model, using a mean-field approximation consistent with the continuum model. The resulting lattice model is determined by what we term the "implicit canonical measure," the most probable distribution on the discrete phase space subject to those constraints. We have proved that this measure has a concentration property with respect to the microcanonical constraint manifold at $N \rightarrow \infty$. This property, combined with the demonstrated Liouville property for the discretized dynamics, constitutes a rigorous justification of our model.

To the extent that comparisons between our model and numerical studies of magnetofluid turbulence are meaningful, we have found a rather 
striking agreement. The detailed studies of 2D magnetofluid turbulence by Biskamp et al., ${ }^{(5.7)}$ in particular, support the conclusions of our model in several respects. First, these simulations confirm the Gaussianity of the local fluctuations in the magnetic and velocity fields. Second, they verify a direct cascade of energy to small scales and an inverse cascade of (quadratic) flux to large scales. While these nonequilibrium cascades have no place in our equilibrium model, they are strongly indicative of the formation of macroscopic coherent structures and they support the separationof-scales hypothesis on which our continuum model is built. Third, the simulations clearly display the relaxation of the ratio of magnetic to kinetic energy to an equilibrium value greater than 1 , even for small initial ratios. This saturation effect is a quantitative prediction of the statistical equilibrium model.

The model also captures the qualitative behavior of the coherent states over the range of admissible energy values. For small energy values, the simulations of Biskamp et al. reveal a dominant, large-scale structure in the mean magnetic field, which forms through the process of quasistatic coalescence of flux tubes. For large values of initial energy, on the other hand, a typical state resembles homogeneous turbulence in the sense that fluctuations predominate. Our analysis of the statistical equilibrium states has exhibited similar qualitative behavior in these regimes. Indeed, when $E^{\prime \prime}$ is close to its minimum possible value, achieved at the deterministic equilibrium, the equilibrium state consists of a macroscopic mean state (a magnetic island with flow) together with small-variance fluctuations. On the other hand, when $E^{0}$ is much greater than the minimum possible energy, the variance of the fluctuations is correspondingly large, and so the equilibrium state is dominated by turbulence.

In a similar manner, the model predicts the dependence of the ideal turbulent state on the value of cross-helicity relative to energy. We have shown that the proportionality between the mean fields $\bar{B}$ and $\bar{V}$ is identical with the $B-V$ correlation. For small cross-helicity values, the equilibria are nearly magnetostatic, and the model becomes a perturbation of fluxconserving equilibrium theory. ${ }^{(24)}$ For large cross-helicity values, however, the mean field is nearly equipartitioned into magnetic and kinetic parts, and the fluctuations in $B$ and $V$ are highly correlated. This result explains the $B-V$ alignment effect observed in numerical simulations. ${ }^{(36)}$

There are two aspects of the model and its discretization that we have not addressed. First, it would be theoretically attractive to construct a discrete dynamics that conserves $M$ quantities exactly for finite $N$, with $M \rightarrow \infty$ as $N \rightarrow \infty$. Instead, our model imposes approximate dynamical constraints whose truncation errors go to zero as $N \rightarrow \infty$. In this respect, our approach to discretization is similar to the "pseudo-spectral method,"(9) 
which is often used to compute the microscopic evolution. Second, it would be interesting to build lattice models which approach the continuum model asymptotically as $N \rightarrow \infty$, but which have nonvanishing spatial correlations for finite $N$. A model of this kind might give a more realistic picture of the turbulent relaxed state for a slightly dissipative magnetofluid. Among such models, our lattice model would be merely the simplest discretization of the continuous statistical equilibrium problem.

\section{ACKNOWLEDGMENT}

The authors express their thanks to David Kinderlehrer and Craig Zirbel for helpful comments and suggestions. We are grateful to Dieter Biskamp for permitting us to use Fig. 1. We also thank a referee who made many insightful remarks which led to substantial improvements in the manuscript. The work of $R$. J. was partially supported by ARO and NSF through grants to the Center for Nonlinear Analysis, and that of B. T. was partially supported by NSF grant DMS-9307644.

\section{REFERENCES}

1. R. Balescu. Equilibrium and Nonequilibritum Statistical Mudumics (Wiley, New York, 1975).

2. R. Balian. From Microphysics to Macrophysics, I (Springer-Verlag. Berlin, 1991 ).

3. P. Billingsley, Probability and Measure (Wiley. New York, 1986).

4. D. Biskanip, Nonlinear Magnetohydrodynamics (Cambridge University Press, Cambridge. 1993).

5. D. Biskamp and H. Welter, Dynamics of decaying two-dimensional malgnetohydrodynamic turbulence, Phys. Fluids B 1:1964 (1989).

6. D. Biskamp and H. Welter. Magnetic tield amplitication and saturation in two-dimensional magnetohydrodynamic turbulence. Phys. Fluids B 2:1787 (1990).

7. D. Biskamp, H. Welter, and M. Walter., Statistical properties of two-dimensional magnetohydrodynamic turbulence, Phys. Fluids $B$ 2:3024 (1990).

8. M. E. Brachet. M. Meneguzzi, H. Politano, and P. L. Sulem, The dynamics of freely decaying two-dimensional turbulence. J. Fluid Mech. 194:333 (1988).

9. C. Canuto. M. Y. Hussain. A. Quateroni, and T. Zang. Spectral Meahods in Flaid Dymamics (Springer-Verlidg, New York, 1985).

10. A. J. Chorin. Fomicily amd Twhulence (Springer-Verlag, New York, 1994).

11. R. S. Ellis, Entrum! Large Dethatoms ant Statistical Methaniss (Springer-Verlag. New York, 1985).

12. A. Eydeland, J. Spruck, and B. Turkington, Multi-constrained variational problems of eigenvalue type: New formulations and algorithms. Math. Comput. 55:509 (1990).

13. D. Fyle and D. Montgomery, High-beta turbulence in two-dimensional magnetohydrodynamics. J. Plasma Phys. 16:181 (1976).

14. A. V. Gruzinov, Gatussian free turbulence: Structures and relaxation in plasma models. Comments. Plasma Phis. Controlled Fusion 15:227 (1993).

15. D. Holm, J. Marsden. T. Ratiu, and A. Weinstein, Nonlinear stability of lluid and plasma equilibria, Ret. Mod. Phys. 123:1 (1985). 
16. A. D. Ioffe and V. M. Tihimirov, Theory of Exremal Problems (North-Holland. Amsterdam, 1979).

17. M. B. Isichenko and A. V. Gruzinov, Isotopological relaxation, coherent structures, and Gaussian curbulence in two-dimensional magnetohydrodynamics. P/11s. Plasmas 1:1801 (1994).

18. E. T. Jaynes, Information theory and statistical mochanics, Phrs. Rer. 106:620 (1957).

19. R. Jordan. A statistical equilibrium model of coherent structures in magnetohydrodynamics. Nonlinearity 8:585 (1995).

20. R. Jordan and B. Turkington. Maximum entropy states and coherent structures in magnetohydrodynamies, in Maximum Entrop! aml Batesian Mothods (Dordrecht, Kluwer, to appear).

21. R. Kinney, J. C. McWilliams, and T. Tajima, Coherent structures and urbulent cascades in two-dimensional incompressible magnetohydrodynamic turbulence. Phis. Plasmas 2:3623 (1995).

22. R. H. Kraichnan and D. Montgomery, Two-dimensional turbulence, Rep. Prog. Phỵs. 43:547 (1980).

23. T. D. Lee, On some statistical properties of hydrodynamical and magnetohydronamical lields. O. Appl. Math. 10:69 (1952).

24. A. E. Litshitz, Magnetohydrodynamics and Spectral Theory (Kluwer, Dordrecht 1989).

25. D. Lynden-Bell, Statistical mechanics of violent relaxation in stellar systems, Monthly. Nolices R. Astron. Soc: 136:101 (1967).

26. J. C. McWilliams, The emergence of isolated, coherent vortices in turbulent llow. J. Fluid Mech. 146:21 (1984).

27. J. Miller, Statistical mechanics of Euler equations in two-dimensions, Phys. Ret Le't. 65:2137 (1990).

28. J. Miller, P. B. Weichman, and M. C. Cross, Statistical nechanics, Euler's equation, and Jupiter's Red Spot, Phy's. Ret. A 45:2328 (1992).

29. D. Montgomery, L. Turner, and G. Vahala. Most probable states in magnetohydrodynamics. J. Plasma Phys. 21:239 (1979).

30. L. Onsager, Statistical hydrodynamics, Nuono Cimente 6:279 (1949).

31. H. Politano, A. Pouquet, and P. L. Sulem, Inertial ranges and resistive instabilities in twodimensional magnetohydrodynamic turbulence, Phax. Fluith $B$ 1:2330, (1990).

32. R. Robert, A maximum entropy principle for two-dimensional perlece fluid dynanics. J. Stat. Pints. 65:531 (1991).

33. R. Robert and 1. Sommeriz. Statistical equilibrium stutes for two-dmensional Tows. J. Fluid Mech. 229:291 (1991).

34. J. V. Shebalin, Broken ergodicity and coherent structure in homogeneous turbulence. Physica D 37:173 (1989).

35. J. Stoer and R. Bulirsch. Introduction to Numerical Amulysis (Springer-Verlag. New York. 1980).

36. A. C. Ting. W. H. Matthateus, and D. Montgomery. Turbulent relixation processes in magnetohydrodynamics. Phys. Fluids 29:3261 (1986).

37. B. Turkington and $R$. Jordan, Turbulent relaxation of a magnetolluid: A statistical equilibrium model, in Adrances in Geomeric Analysis and Continum Mechanics (International Press, Boston, 1995). pp. 124-137.

38. B. Turkington, A. Lilshitz. A. Eydeland, and J. Spruck, Multiconstrained variational problems in magnetohydrodynamics: Equilibrium and slow evolution, J. Comput. Phys. 106:269 (1993).

39. L. Woltjer, Hydromagnetic equilibriums III, Astrophys. J. 130:400 (1959). 